Perez, C. \& Arroyo, P. (2019). "Designing Municipal Waste Management Programs Using Choosing by Advantages and Design Structure Matrix." In: Proc. $27^{\text {th }}$ Annual Conference of the International. Group for Lean Construction (IGLC), Pasquire C. and Hamzeh F.R. (ed.), Dublin, Ireland, pp. 1345-1368. DOI: https://doi.org/10.24928/2019/0194. Available at: 〈www.iglc.net>.

\title{
DESIGNING MUNICIPAL WASTE MANAGEMENT PROGRAMS USING CHOOSING BY ADVANTAGES AND DESIGN STRUCTURE MATRIX
}

\author{
Catalina Perez ${ }^{1}$ and Paz Arroyo ${ }^{2}$
}

\begin{abstract}
Designing public environmental policies is not an easy task. Decision makers must consider multiple social, environmental and economic aspects in order to achieve sustainable solutions. They must also manage interrelated information and preferences of different stakeholders to ensure that the policy suits the community and accomplishes its goals. This research study focused on analyzing the design process of environmental public policies using the Choosing by Advantages (CBA) decision system integrated with Design Structure Matrix (DSM) to make complex decisions. A case study involving the redesign of a waste collection program was conducted, where three complex decisions were made using the CBA method. Researchers proposed using DSM to decrease negative iterations by finding the optimal order of decisions. Using DSM helped to decrease the number of iteration loops, and document the assumptions to make the process more transparent. Future work should measure the impacts of using CBA together with DSM to make decisions in the formulation process of public environmental policies.
\end{abstract}

\section{KEYWORDS}

Waste, choosing by advantages, design structure matrix, negative iteration, sustainability

\section{INTRODUCTION}

Designing a new policy related to environmental management is a difficult task for decision-makers due to the amount of information and the involvement of multidisciplinary fields (Buzuku, Kraslawski, \& Kässi, 2016). Moreover, it is necessary to include citizens and the community's visions about the program during the planning and design stages (Renn, 2006). In particular, Municipal Solid Waste (MSW) management is a complex problem that addresses multi-trajectory, non-linear, and dynamic interrelated components

1 Master Student, Department of Construction Engineering and Management, Pontificia Universidad Católica de Chile.

2 DPR Quality Leader, San Francisco, CA, PazA@dpr.com. and Adjunct Professor, Department of Construction Engineering and Management, Pontificia Universidad Católica de Chile. 
of a system that must consider different aspects such as social, economic, and environmental dimensions to achieve its goals (Hoornweg \& Bhada-Tata, 2012).

The complexity of designing waste collection programs also lies in the many variables that must be addressed, where every variable has several options associated with it. Decision-makers must put special emphasis on the design stage since variables design directly impacts the effectiveness of the program and its participation rate (Renn, 2006).

Traditional practices cannot define and describe the complexity of the problem in early stages of the design process because of its uncertainty (Buzuku, Kraslawski, \& Harmaa, 2015). Moreover, these practices usually lack the capability to perform sustainability analyses in early stages of design development. All these failures result in an inefficient process of retroactive modifications to the final solution (Azhar, Carlton, Olsen, \& Ahmad, 2011).

Incorporating conflicting factors with different criteria is required in order to assess possible solutions for sustainability problems. Using multicriteria approaches is needed to support the decision-making process during the design stage. This approach must also be capable of managing stakeholders' preferences and including the community's visions as inputs to make key decisions about the features of the policy. Moreover, designers must consider and manage the interrelated decisions and information that the design process creates, in order to discover the real effects and impacts that the problem generates (Liew \& Sundaram, 2009).

The purpose of this study is to establish a systematic approach to design public environmental policies related to solid waste management that consider and manage interrelated decisions and information created during the process. It also allows for the management of stakeholders' preferences and community visions during the design stage. In this context, this paper focuses on the synthesis of methods and techniques such as Choosing by Advantages (CBA) decision system and Design Structure Matrix (DSM) to allow for the management of stakeholder preferences to support interrelated decisionmaking processes. Researchers conducted a case study where three decisions on redesigning a curbside waste collection program were analyzed. The proposed approach seeks to clarify the decision-making process and avoid negative iterations that are activities and work that do not add value to the final process (Ballard, 2000).

\section{LITERATURE REVIEW}

\section{Municipal Solid Waste Management}

MSW Management has become a problem since the global impacts of solid waste are growing fast. Among these impacts are high levels of GHG emissions, large occupation of landfill spaces, and contributions to flooding and air pollution that affect public health (Hoornweg \& Bhada-Tata, 2012). The global nature of MSW also involves increasing number of links between products, urban practices, and the recycling industry. Therefore, choosing appropriate technology and methods to manage all the diversity encompassed within MSW helps to improve human health and environment, promote reuse and recycling, enhance waste prevention programs, and implement extended producer responsibility collectively (Pires, Martinho, \& Chang, 2011). 
The complexity and uncertainty of environmental problems arise from the difficulty of addressing social, environmental, and economic dimensions, and from insufficient knowledge presented in the design stage. These impose a need for integration of additional information as it becomes available, and call for decision-making processes that allow for integration of different value judgement and logics (Hove, 2000).

For waste management policies, their effectiveness relies on participation rates, and it is therefore crucial to consider the citizens' preferences during the design process (Renn, 2006). Policy-makers and experts must also participate in order to address different aspects to design for sustainability. Consequently, practitioners need to have a shared understanding of the interactions and interdependencies between social, economic, and environmental goals, their complementarities, and potential policy conflicts and trade-offs (OECD, 2016) in order to design an effective policy focused on sustainable development.

On one hand, the formulation of new environmental public policies implies a-reconceptualizing the role of experts, practitioners, and the community in the production and use of information to support the whole process. Therefore, the formulation process needs a system to manage stakeholders' preferences and support a participatory process. On the other hand, sustainable solutions also demand a re-configuration of complex sociotechnical systems (Adrian \& John, 2009), thus policy makers must emphasize their consideration of all interrelated information to discover the true impacts of the final policy.

\section{INTERRELATED DECISION-MAKING PROCESS}

A multicriteria decision making (MCDM) approach is needed to address high uncertainty, conflicting objectives, multiple stakeholders and their preferences, and different aspects of sustainability (Wang et al., 2009). There are several MCDM methods widely used in design processes such as Weighting rating and calculating (WRC) and Analytical hierarchy process (AHP). However, not all of them can manage the decision-making process in a sustainable manner. Studies that compare WRC and AHP with Choosing by Advantages (CBA) decision system showed that CBA performs better than the others when making sustainable decisions (Arroyo et al., 2015), because the other methods do not consider relevant differences between the alternatives. Additionally, weighting factors are used to hide the trade-offs of the decisions, and thus decision-makers do not fully understand the implication of each criteria. Moreover, CBA is capable of creating a transparent and collaborative environment for making sound decisions in design (Kpamma et al., 2016).

There are several studies which formulate frameworks, guidelines, and assessment approaches to improve the involvement of stakeholders in the design of public policies (Edelenbos \& Klijn, 2006; Thabrew, Wiek, \& Ries, 2009). However, none of them manage the stakeholders' preferences and deliver value through a decision-making system. Kpamma et al. (2016) used CBA to manage the preferences of users during design stage in the construction industry. As a result, CBA worked as a participative decision method that promoted a collaborative environment among stakeholders. CBA also fostered an atmosphere that enabled knowledge sharing and common understanding of the information created in the design process.

Nevertheless, CBA cannot consider the interrelation of the decisions. Disregarding these interrelations hide the real effects and impacts that the problem generates in the 
project's lifecycle (Liew \& Sundaram, 2009). With this context in mind, researchers propose using Design Structure Matrix (DSM) to manage interrelated information and decisions. DSM is a tool that allows the user to analyze projects or models with the purpose of decomposing and integrating problems (Browning, 2001). This is achieved by breaking down a system into its elements, tasks, or decisions, understanding and documenting the interrelations of these tasks, and analyzing potential reintegration of them via clustering or sequencing. Resequencing activities aims to identify coupling in the process, thus allowing the design team to try solving iterations problems, isolate uncertainty, and raise the confidence associated with decision-making (Eppinger, Whitney, Smith, \& Gebala, 1994). In addition, reorganizing information helps maximize the availability of information, minimize the size of iteration loops, and minimize the number of iterations. There are several studies that focus on applying DSM in the design stage (e.g. Browning, 2001; Pektaş \& Pultar, 2006; Tuholski \& Tommelein, 2010). All studies had positive impacts on the project's outcomes. DSM helped to document and understand activity dependencies, share available information in early stages of design, put effort into coordination, and expose conflicts due to the interrelation of tasks and planning iterations to reduce wastes. Other studies provide information flow models through DSM (Baldwin et al., 1999; Eppinger et al., 1994) to manage the design process. There are few studies that relate DSM with sustainable policy formulation (Buzuku et al., 2015, 2016). However, these studies hide the trade-offs of the different possible solutions by optimizing the whole system behind criteria that are not necessarily understood by all stakeholders. This study proposes using DSM to identify interrelated decisions, then resequencing them to avoid negative iteration during the design stage. Additionally, it aims to separate the entire problem into approachable tasks, and reintegrate it to define the decision-making process.

\section{RESEARCH METHOD}

This research uses a case study to analyse the decision-making process using the CBA method to make decisions regarding the design of municipal solid waste management policies and programs. The case study protocol was: 1) The design team made decisions using CBA decision system, 2) Researchers collected practitioners' feedback about the process to understand how CBA worked for complex decisions, and asked the design team to evaluate CBA compared to their previous experiences, 3) Researchers proposed the use of DSM to improve the process by addressing interrelated decisions and information. 4) With the support of the design team, researchers found the optimal order of decisions to avoid negative iteration in the design process. Finally, 5) researchers compared the design process using CBA together with DSM vs. traditional practices where no formal method was used.

\section{CASE STUDY}

The project adopted for the case study was the redesign process of a waste collection program for a city with a population of approximately 275.000 . The scope of the program included evaluating redesigning the existing recycling program, designing an organic waste collection program, evaluating redesigning the program of waste collection, defining 
financing options, cart sizes, depots, and collection frequencies for the different programs. Specific tasks considered in the process included the development of a variable rate waste utility (PAYT Utility) and evaluation of depots and other waste services.

The main decisions: defining the Recycling Program, Organics Program, and PAYT Utility were made using three separate CBAs. To create alternatives and to define the order of the decisions in the early stages of the design process, the team created a decision tree to visualize and define the information flow. To decide on the list of Factors, multiple stakeholders were consulted by one project manager for each CBA. The project manager for each decision had to decide which Factors to include and which to consolidate into bundles that represented a single common Factor. The team used CBA for each decision through workshops to facilitate the decision-making consensus. The CBA framework was delegated to specific subject matter experts who then populated the Attributes and Advantages of their assigned Factors. The team also created financial models for each Alternative, and the average initial monthly cost for customers was calculated. Costs for each Alternative were then plotted against the Total Importance of Advantages for each Alternative. To ensure that the redesigned system reflected the preferences of the public, an external consultant was hired to assist with the development of a community engagement process, and the analyzed results were used to populate Attributes and Advantages in order to capture the values of the community in the decisions. Finally, practitioners gave feedback on the process through a survey and interviews in order to better understand the advantages and disadvantages of using CBA for making complex decisions related to public environmental policies.

\section{NEGATIVE ITERATION DURING THE DESIGN PROCESS}

Researchers gathered the practitioners' comments during the workshops, and used them to understand how to approach failures within the process, and how to improve the implementation of CBA. The main problems were the complexity of the decisions, and understanding which factors to assess in order to find a sustainable solution. The most timeconsuming tasks were defining the key decisions to be made before starting a CBA, and defining the factors for every decision. Moreover, the interconnection of information through the process made decisions difficult. CBA is limited due to its disregard for interrelations between the decisions that could bring problems to discover the real impacts of the program due to the amount of information created that is inter-linked in the process (Liew \& Sundaram, 2009). CBA approach makes decisions as isolated systems that could increase the iteration process and increase the time it took to make the decisions. Thus, without a tool that manages all the information, the process needed constant review and rework.

Using supplementary tools that address interrelated information could mitigate the impacts of not considering interrelated decisions. To improve the design process for complex decisions, researchers propose using DSM to separate the whole problem in order to better understand the complexity and uncertainty of the process. Then, DSM can reintegrate the process to have all the information available at the time that is needed. First, it is necessary reintegrate the defined decisions through dependency sequence to discover the optimal order of decisions. This helps to maximize the availability of information, 
minimize the size of iteration loops, and minimize the number of iterations (Austin et al., 2000). Second, a revision of the process must be conducted to understand where the iteration loops and tearing dependencies are, to avoid negative iteration during the design stage. Then, practitioners and decision-makers can see the interrelation of the decisions and consolidate the path the process should follow.

\section{REINTEGRATION VÍA SEQUENCING DEPENDENCY}

Due to the complexity of the decisions and the manual design procedure of the decision tree, it was difficult to see some of the relationships between the decisions. The result was a fuzzy decision tree where the dependencies between decisions were difficult to show in one information flow. The decision tree is presented in Figure 1.

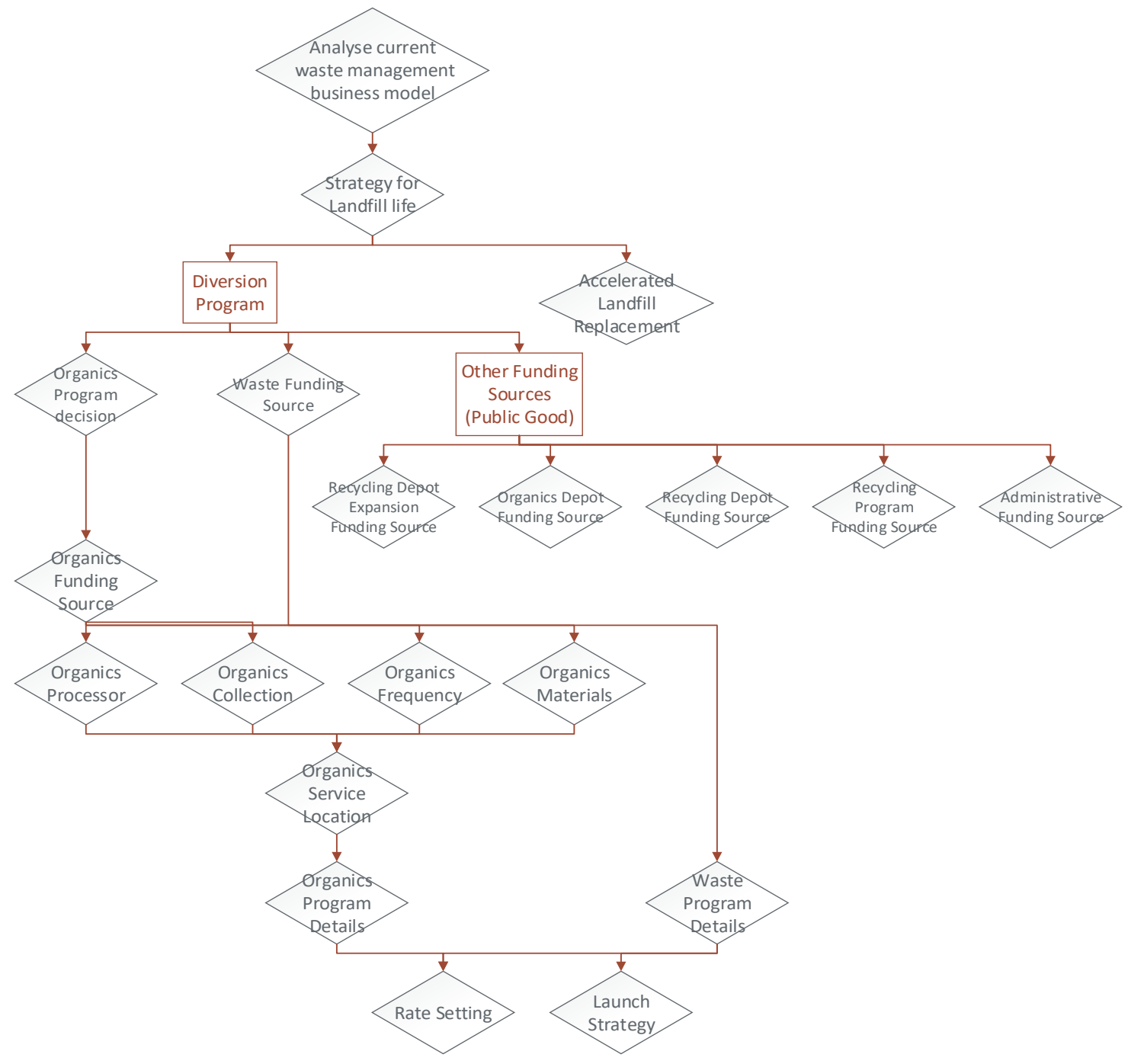

Figure 1: Decision tree of the design process 
The tool presents different paths where the information needed to move to the next cluster is not well defined. Not defining a clear path forward could increase the number of iteration loops and the time it takes to make decisions. Also, this type of tool can model parallel and sequential tasks, but cannot model feedback and iteration that is characteristic of the design stage.

Researchers created a DSM to illustrate the dependencies of the problem and find a path that would minimize the number of iteration loops during the design stage. To create the DSM, researchers considered the decisions to be made in an random order, and assigned the dependencies by consulting with an expert on the design team. These are the unified decisions for the three separate CBAs made for Recycling, Organics, and PAYT programs. Decisions to analyze and their dependencies are shown in Figure 2.

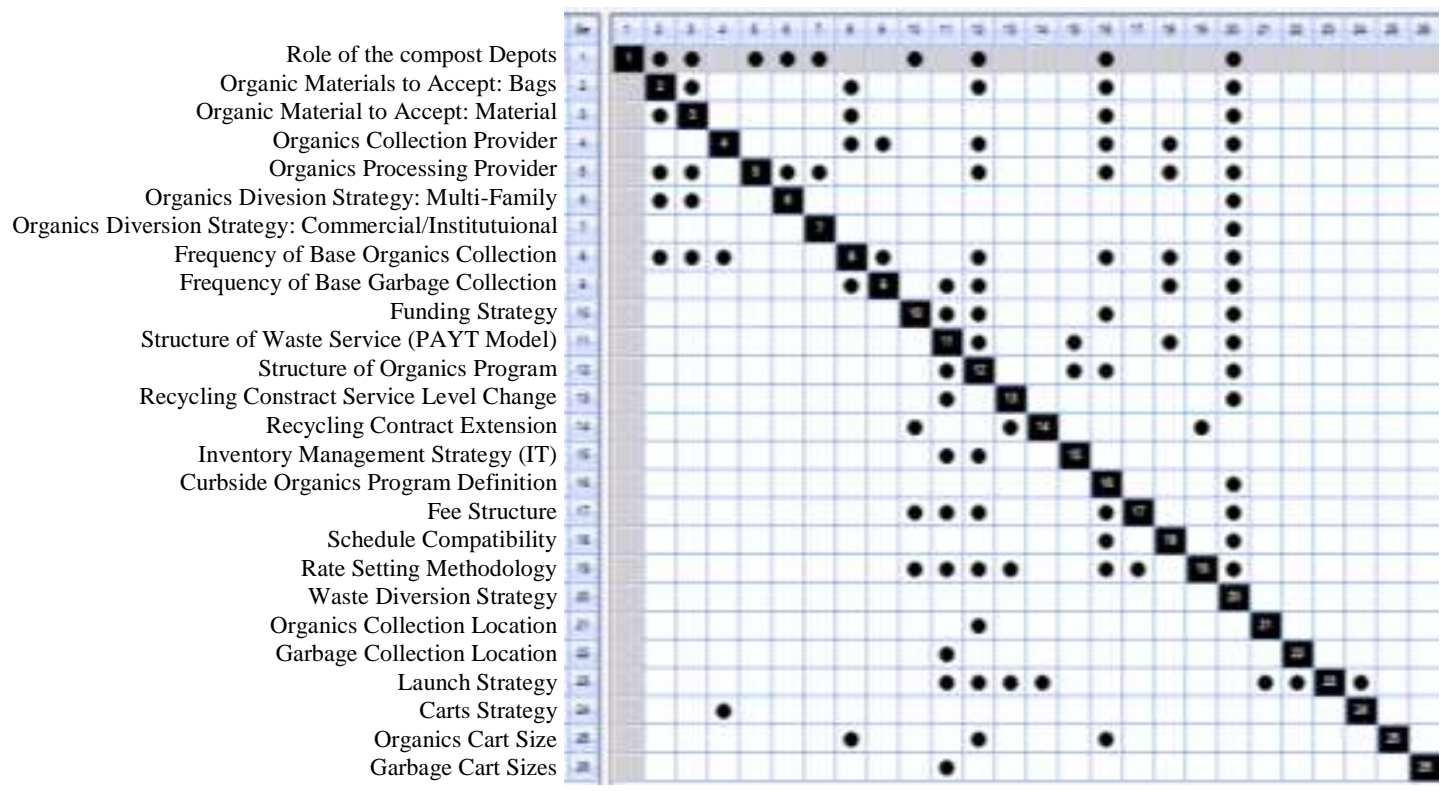

Figure 2: DSM for unified waste utility decisions for a waste collection program

\section{TEARING AND REVIEWING DSM}

DSM can reintegrate the decisions through dependency sequence to find the optimal order according the dependencies set before. Figure 3 shows the results of DSM before and after revision (Figure $3 \mathrm{a}$ and $3 \mathrm{~b}$ respectively). The first iteration of DSM shows two iteration loops. The first loop involves decisions regarding making changes to the Inventory Management Strategy (IT), defining the Structure of the PAYT Model, and defining the Structure of the Organics Program. To solve this problem, the dependencies of making changes to the IT Strategy were teared because there was not sufficient time to implement comprehensive changes to the existing IT Strategy according to the results of the other decisions. After this tearing, only decisions regarding the structure of the Organics Program and the PAYT Model are interrelated. Decision-makers can put effort into designing a 
solution were the two decisions have an optimal performance together. The other iteration loop involved the following decisions: (1) Define role of bags for the Organics Program; (2) Define accepted Materials for the Organics Program; (3) Define Collection Provider for the Organics Program; (4) Define frequency of Organics Collection; and (5) Define Frequency of Garbage Collection. To solve this iteration loop, three assumptions were made before making the decisions. First, in relation with Organics Collection Frequency, the assumptions set were choosing the alternative with lowest cost regardless of interdependent impacts, and tearing the dependency with the definition of Organics' carts size. Second, to solve the interdependencies of Garbage Collection Frequency, the alternative with lowest cost was chosen. Finally, to solve the interdependencies with defining which Material to accept in the Organics program, the decision was made using a separate and less complex CBA that no plastic would be accepted. Tearing these dependencies changes the size of the iteration loops where only two decisions are interrelated in every loop. It helps to have all information available when these decisions are made. Moreover, by illustrating the information flow and marking where the iteration is could prepare practitioners to make interrelated decisions and know what information is needed to make these decisions.

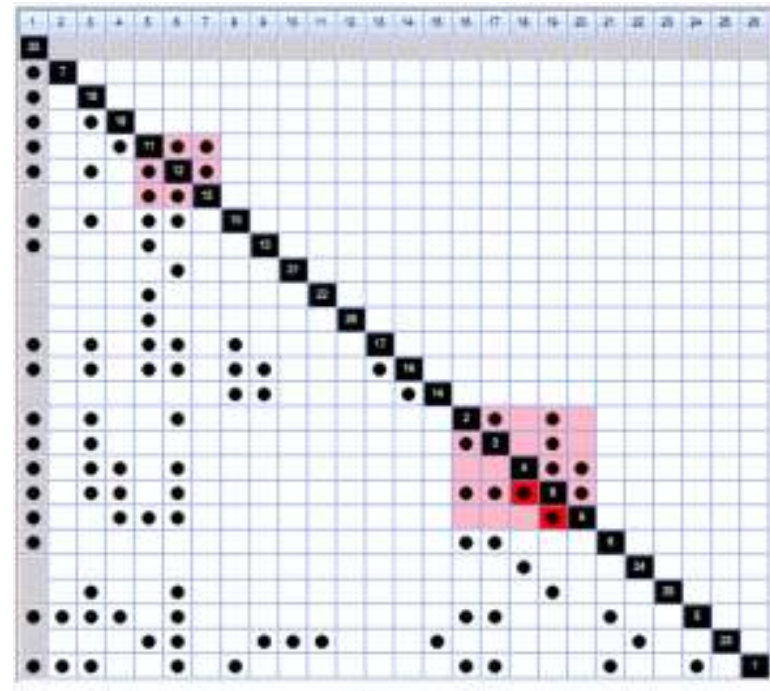

(a)

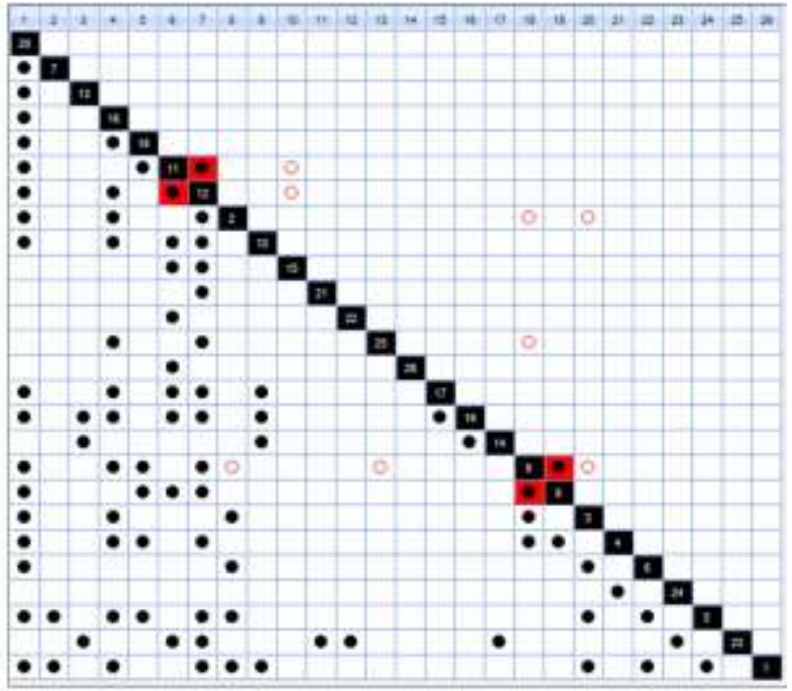

(b)

Figure 3: (a) First result of DSM (b) DSM results after revision

\section{DISCUSSION}

Using DSM together with CBA allows the user to make interrelated decisions and managing stakeholders' preferences. Additionally, integrating DSM and CBA allows for the documentation of interdependencies among decisions in a fast and simple manner. It also allows for the manipulation of high complexity levels in contrast to traditional tools as decision trees. Therefore, this method would enable practitioners to handle complex problems as a whole, as opposed to traditional practices where interdependencies are not clear for all stakeholders. In addition, DSM permits the documentation of assumptions 
required to move forward along a linear/sequential path. This makes the process more transparent by registering information that would serve to review the process for an eventual policy modification and for creating reports for the accountability process delivered to the community and the governance. Moreover, the method identifies the optimal order of decisions by not influencing the sequence based on undocumented knowledge. Thus, all information that is needed to make decisions is documented and understood for all stakeholders that participate in the process.

There are other applications of DSM integrated with CBA that can solve problems of negative iteration, but are not discussed in this work. Clustering the factors that practitioners must assess could decrease the time it takes to define the factors of the CBAs. This analysis could avoid excessive time in the definition of the condensed factors by nreaking down the problem. Then, a reintegration of the factors via clustering sequence could avoid duplicated factors and improve the consensus on what practitioners should evaluate when choosing the best alternative to meet the goals of the program.

\section{CONCLUSIONS}

This work contributes to the body of knowledge by using CBA together with DSM for make complex decisions to formulate environmental public policies. In particular, three decisions were made to redesign a waste collection program. CBA allowed practitioners to assess multiple aspects in Organics, PAYT Utility, and Recycling Program decisions to achieve sustainable solutions. Preferences of the community and municipality were included in the assessment, along with environmental and economic factors to ensure that the program suited the features of the community and accomplished the goals of the governance. Nevertheless, CBA is limited due to the fact that it does not consider interrelated decisions that impact the process as an excessive time in the definition of the decisions and Factors. To solve this problem, researchers proposed using DSM to find the optimal order of decisions to decrease the size of the iteration loops and decrease negative iteration during the process. The method proposed helped to find the optimal order of decisions according to their dependencies, document assumptions to move forward along a linear path, and identify iterative loops of decisions. Then, practitioners and decisionmakers could put forth special effort in finding optimal solutions for two decisions without disregarding their interrelation. The limitation of the study resides in that the results of the implementation of CBA and DSM are based on interviews using a case study, thus its impacts in terms of time or cost savings are not possible to evaluate accurately. Future work should measure the impact of using CBA together with DSM to know if negative iteration decreases during the design stage. Future work should also test using DSM to clustering Factors and define them without constant rework, especially for complex decisions where factors are interdependent.

\section{ACKNOWLEDGMENTS}

Researchers would like to thank the decision making team and practitioners that participated in the CBA workshops and helped to evaluate the design process. All their support is gratefully acknowledged. Any opinions, findings, conclusions or 
recommendations expressed in this paper are those of the authors and do not necessarily represent the views of the people involved in the design process

\section{REFERENCES}

Arroyo, P., Tommelein, I. D., \& Ballard, G. (2012). Comparing Multi-Criteria DecisionMaking Methods to Select Sustainable Alternatives in the AEC Industry. Icsdec 2012, 869-876. https://doi.org/10.1061/9780784412688.104

Arroyo, P., Tommelein, I. D., \& Ballard, G. (2015). Comparing AHP and CBA as Decision Methods to Resolve the Choosing Problem in Detailed Design. Journal of Construction Engineering and Management, 141(1), 04014063. https://doi.org/10.1061/(ASCE)CO.1943-7862.0000915

Austin, S. A., Baldwin, A. N., Baizhan, L. I., \& Waskett, P. (2000). Analytical design planning technique (ADePT): A dependency structure matrix tool to schedule the building design process. Construction Management and Economics, 18(2), 173-182. https://doi.org/10.1080/014461900370807

Azhar, S., Carlton, W. A., Olsen, D., \& Ahmad, I. (2011). Building information modeling for sustainable design and LEED®rating analysis. Automation in Construction, 20(2), 217-224. https://doi.org/10.1016/j.autcon.2010.09.019

Baldwin, A. N., Austin, S. A., Hassan, T. M., \& Thorpe, A. (1999). Modelling information flow during the conceptual and schematic stages of building design. Construction Management and Economics, 17(2), 155-167. https://doi.org/10.1080/014461999371655

Browning, T. R. (2001). Applying the design structure matrix to system decomposition and Inintegration problems: a review and new directions. IEEE Transactions on Engineering Management, 48(3), 292-306. https://doi.org/10.1109/17.946528

Buzuku, S., Kraslawski, A., \& Harmaa, K. (2015). Supplementing Morphological Analysis with a Design Structure Matrix for Policy Formulation in a Wastewater Treatment Plant. Modeling and Managing Complex Systems. Carl Hanser Verlag GmbH \& Co. KG. https://doi.org/https://doi.org/10.3139/9783446447264.002

Buzuku, S., Kraslawski, A., \& Kässi, T. (2016). A Case Study in the Application of Design Structure Matrix for Improvement of Policy Formulation in Complex Industrial Wastewater Treatment. International Dependency and Structure Modeling Conference (Dsm), (August). https://doi.org/10.19255/JMPM-DSM2016

Edelenbos, J., \& Klijn, E.-H. (2006). Managing Stakeholder Involvement in Decision Making: A Comparative Analysis of Six Interactive Processes in the Netherlands Managing Stakeholder Involvement in Decision Making : A Comparative Analysis of Six Interactive Processes in the Netherlands. Journal of Public Administration Research and Theory, 16(3), 417-446.

Eppinger, S. D., Whitney, D. E., Smith, R. P., \& Gebala, D. A. (1994). A model-based method for organizing tasks in product development. Research in Engineering Design, 6(1), 1-13. https://doi.org/10.1007/BF01588087

Hoornweg, D., \& Bhada-Tata, P. (2012). What a Waste: A Global Review of Solid Waste Management. In Urban Development Series; knowledge papers no.15, World Bank (p. 116). https://doi.org/10.1111/febs.13058 
Hove, S. van den. (2000). Participatory approaches to environmental policy-making : the European Commission Climate Policy Process as a case study. Ecological Economics, 33, 457-472.

Kpamma, Z. E., Adinyira, E., Ayarkwa, J., \& Adjei-Kumi, T. (2016). Application of the CBA Decision System to Manage User Preferences in the Design Process. Journal of Professional Issues in Engineering Education and Practice, 142(1), 05015004. https://doi.org/10.1061/(ASCE)EI.1943-5541.0000258

Liew, A., \& Sundaram, D. (2009). Flexible modelling and support of interrelated decisions. Decision Support Systems, 46(4), 786-802. https://doi.org/10.1016/j.dss.2008.11.016

Pektaş, S. T., \& Pultar, M. (2006). Modelling detailed information flows in building design with the parameter-based design structure matrix. Design Studies, 27(1), 99122. https://doi.org/10.1016/j.destud.2005.07.004

Pires, A., Martinho, G., \& Chang, N. (2011). Solid waste management in European countries : A review of systems analysis techniques. Journal of Environmental Management, 92(4), 1033-1050. https://doi.org/10.1016/j.jenvman.2010.11.024

Renn, O. (2006). Participatory processes for designing environmental policies. Land Use Policy, 23, 34-43. https://doi.org/10.1016/j.landusepol.2004.08.005

Thabrew, L., Wiek, A., \& Ries, R. (2009). Environmental decision making in multistakeholder contexts : applicability of life cycle thinking in development planning and implementation. Journal of Cleaner Production, 17, 67-76. https://doi.org/10.1016/j.jclepro.2008.03.008

Tuholski, S. J., \& Tommelein, I. D. (2010). Design Structure Matrix Implementation on a Seismic Retrofit. Journal of Management in Engineering, 26(3), 144-152. https://doi.org/10.1061/(ASCE)ME.1943-5479.0000016

Wang, J., Jing, Y., Zhang, C., \& Zhao, J. (2009). Review on multi-criteria decision analysis aid in sustainable energy. Renewable and Sustainable Energy Reviews, 13, 2263-2278. https://doi.org/10.1016/j.rser.2009.06.021 
Perez, C. \& Arroyo, $P$ 